\title{
Quantifying spatial correlations of fluorescent markers using enhanced background reduction with protein proximity index and correlation coefficient estimations
}

\author{
Vadim Zinchuk ${ }^{1}$, Yong $\mathrm{Wu}^{2}$, Olga Grossenbacher-Zinchuk ${ }^{3}$ \& Enrico Stefani ${ }^{2}$ \\ ${ }^{1}$ Department of Anatomy and Cell Biology, Kochi University, Faculty of Medicine, Kochi, Japan. ${ }^{2}$ Department of Anesthesiology, Division of Molecular Medicine, \\ David Geffen School of Medicine, University of California at Los Angeles, Los Angeles, California, USA. ${ }^{3}$ Unit of Anatomy, Faculty of Medicine, University of Fribourg, \\ Fribourg, Switzerland. Correspondence should be addressed to V.Z. (zinchuk@kochi-u.ac.jp).
}

\begin{abstract}
Interactions of proteins are examined by detecting their overlap using fluorescent markers. The observed overlap is then quantified to serve as a measure of spatial correlation. A major drawback of this approach is that it can produce false values because of the properties of the image background. To remedy this, we provide a protocol to reduce the contribution of image background and then apply a protein proximity index (PPI) and correlation coefficient to estimate colocalization. Background heterogeneity is reduced by the median filtering procedure, comprising two steps, to reduce random noise and background, respectively. Alternatively, background can be reduced by advanced thresholding. PPI provides separate values for each channel to characterize the contribution of each protein, whereas correlation coefficient determines the overall colocalization. The protocol is demonstrated using computer-simulated and real biological images. It minimizes human bias and can be universally applied to various cell types in which there is a need to understand protein-protein interactions. Background reductions require 3-5 min per image. Quantifications take <1 min. The entire procedure takes approximately 15-30 min.
\end{abstract}

\section{INTRODUCTION}

The interaction of proteins via complex networks is one of the fundamental principles of cell and molecular biology and is the basis for the dynamic control of cellular metabolism. Proteins interact by direct binding, modification or by acting on a substrate and converting it. Binding of molecules to each other increases with the respective increase in their local concentrations, and it can be amplified when/if they are colocalized. In addition, major regulatory proteins are usually 'switchable' and can be modulated by other molecules following their contact ${ }^{1}$. Thus, understanding their proximity and possible contact may have a crucial role in envisioning their impact in signal transduction.

Methodologically, the interaction of proteins can be studied with the help of various techniques, some of which are based on the use of confocal fluorescent microscopy. Because resolution of microscopes is limited, proteins are detected indirectly by labeling them by two (most frequently) or more differently colored fluorophores with emission at distinctive wavelengths. Thereafter, additional techniques, such as fluorescence energy transfer, can be applied to identify the transfer of energy between fluorophores, thereby suggesting interaction of the respective proteins ${ }^{2}$. A decisive observation in these studies is the existence of overlap of the applied fluorophores as an important proof that the respective proteins are indeed located closely enough to be capable of interaction. Visually, the overlap of the original colors of fluorophores will result in the appearance of a new, third, overlaid color, indicating colocalization. Observation of colocalization in fluorescence microscopy images was a significant advance for cell and molecular biological research ${ }^{3,4}$. Importantly, with the introduction of the ability to estimate the degree of this overlap quantitatively, it became possible to characterize it objectively as well ${ }^{5-7}$. Previous work provided important information about the steps needed to perform quantification of colocalization properly, stressing the importance of: (i) avoiding the effect of bleed-through during acquisition of images; (ii) removing background fluorescence in them; and (iii) using optical sections rather than projections for its analysis ${ }^{3,7,8}$. Because of advances in digital imaging and a substantial increase in the use of fluorescence microscopy to perform spatial and temporal calculations in biology and medicine, deserved attention has been recently paid to the basic procedures of image acquisition, processing and handling to be followed to ensure accuracy of measurements in medico-biological images ${ }^{9}$.

Although the methodology to quantify colocalization of overlapping proteins has been available for over a decade ${ }^{5}$, the crucial issue of background fluorescence in the images being analyzed proved to be one of the most challenging issues to solve. Background comes from a variety of sources, such as components of the sample mounting medium and/or unrelated proteins in the cytoplasm that can fluoresce as well ${ }^{10}$. It contributes to the signal of interest and may eventually camouflage the actual structures to be quantified. To achieve reliable quantification results, background should be reduced as much as possible. It was recently reported that the failure to address the contribution of the background properly can potentially result in up to $30 \%$ overestimation of colocalization ${ }^{11,12}$ and therefore lead to incorrect conclusions. A common way to reduce background is to use a simple thresholding, which removes a certain amount of selected color pixel values from the image $\mathrm{e}^{7}$. However, this technique (which can be done with almost any major imageediting software) is extremely biased and highly controversial, as selection of the number and properties of the pixels to be removed is entirely empirical. A better method is deconvolution, which makes sharper and crisper images. Deconvolution is a softwarebased procedure (software is available from Huygens, Openlab and 
Figure 1 | Flowchart of the procedure for quantification of the spatial correlation of fluorescent markers. The protocol presented here focuses on background reduction and quantification steps. (1) Background in analyzed images can be reduced by applying median filtering with shot noise removal (Steps 1-5). Alternatively, background can be also reduced by the advanced thresholding procedure (Steps 6 and 7). (2) After background reduction, images can be quantified by calculating the values of the PPI (Steps 8-11) and a set of coefficients (Steps 12 and 13). (3) Finally, the results are interpreted according to the distinct values for the PPI and the coefficients used.

others) that restores the optical distortion of the images occurring in optical microscopes by using different point-spread functions (PSFs) and assessing whether it results in improvement of their characteristics ${ }^{13}$. Unfortunately, it also remains flawed because of the doubts raised by many researchers about the eventual accuracy of the quantification of colocalization because of the magnitude of the change the procedure introduces to the images. Another problem is that uncertainty about background conditions in analyzed images forces researchers to use a number of different coefficients (such as the correlation coefficient $(\mathrm{Rr})$, the overlap coefficient $(R)$, the overlap coefficients $k_{1}-k_{2}$ and the colocalization coefficients $m_{1}-m_{2}$ ), which frequently produce contradictory results ${ }^{4}$.

Previously, we reported a protocol in which quantification of colocalization was performed using an advanced thresholding procedure for background reduction with further coefficient calculations ${ }^{7}$. Although the protocol proved to be very useful ${ }^{4}$, it relies on five different coefficients (two independent and two pairs for each image region of interest (ROI), plus one pair for each scattergram $\mathrm{ROI}$ ), which can make it difficult to decide which one of them is best suited to the conditions of a particular experiment. In addition, it is essentially a 2D approach. In the present protocol, we combined the median filtering and advanced thresholding procedures to reduce background with calculations of the protein proximity index (PPI), which is applied to pixels presented as $3 \mathrm{D}$ plots, and a set of coefficients $^{7,14}$. We analyzed various coefficients and determined that $\mathrm{Rr}$ is the most applicable of them. Notably, images processed for background reduction using median filtering can be quantified using not only PPI but also a set of coefficients. Similarly, images processed for background reduction using advanced thresholding can be examined using a set of coefficients as well as the PPI. This approach allows double-checking of calculation results to ensure their reliability. The PPI is used to characterize colocalization of each protein by obtaining separate values of the index for each channel, whereas Rr is used to determine its overall degree. An overview of the protocol as a flowchart is given in Figure 1. To facilitate understanding of the protocol and its adoption by readers with different scientific expertise, we prepared a list of the frequently used terminology with its concise description (Box 1). A major advantage of this protocol is that it minimizes human bias in quantitative detection, and thus substantially improves the applicability of the obtained results.

It should be mentioned that there are several limitations of the technique presented here. First, although PPI values represent the percentage of colocalized molecules in ideal situations, in real settings the calculated values could sometimes be greater than $100 \%$ (1.0). This may occur if images in two channels have very different levels of background. In this case, quantification should rely solely on Rr results. In addition, in some cases in which fluorescent staining is very weak, background reduction procedures may not provide satisfactory results and therefore quantification on

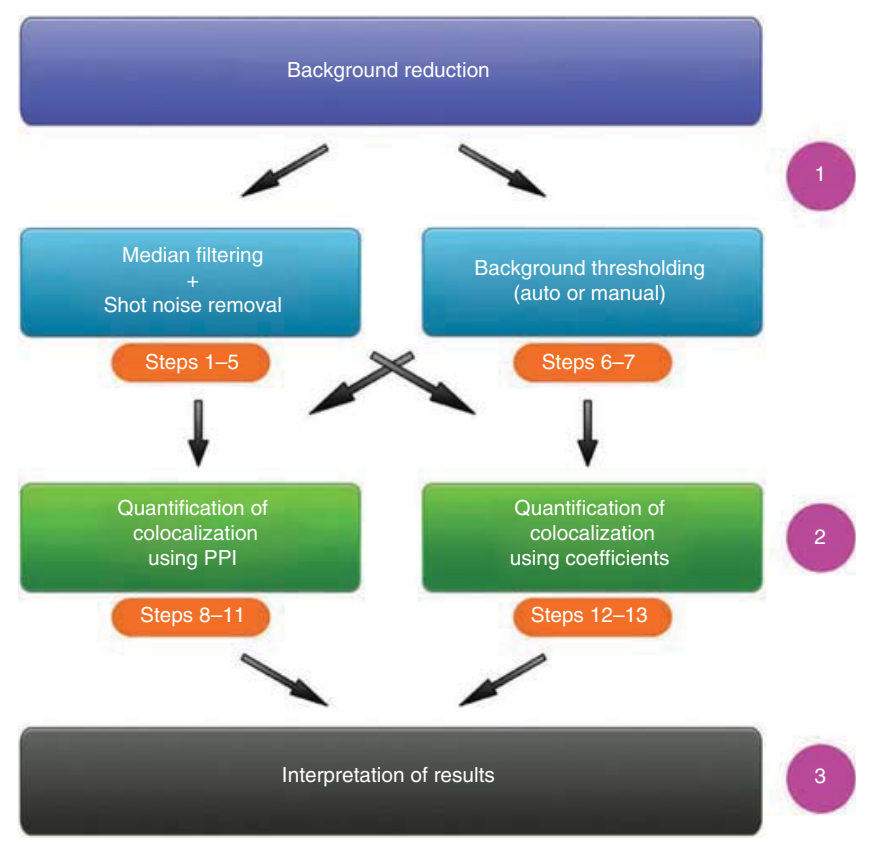

such samples should be considered cautiously. As an indication, the values of calculated coefficients may be out of their standard ranges (see PROCEDURE). In addition, image files saved in the microscope manufacturers' proprietary file formats are not directly usable and should be resaved as lossless TIFF files before importing for analysis.

The protocol can be adapted to various types of cells in which there is the need to understand the interaction of proteins involved in signal transduction. It should be especially useful when studying membrane proteins as receptors and ion channels targeted by drugs. Although the field of microscopy is rapidly advancing, with new methodologies and super-resolution microscopes being introduced ${ }^{15}$, the use of confocal fluorescence microscopy images for studying protein-protein interactions, and thus the described protocol, will likely remain in demand and relevant for some time to come.

\section{Experimental design}

Preparation and processing of images. The protocol assumes that images prepared for analysis were properly acquired and processed and are therefore suitable for quantification ${ }^{3,7}$. You should also ensure that samples were correctly prepared, that the microscope setup included the use of optimized emission filters and the proper pinhole size, and that images were acquired by sequential scanning and saved in original file format ${ }^{8}$. In particular, it is necessary to ensure that the fluorescent antibodies used to capture their respective proteins are specific and do not cross-react, as well as that samples are free of autofluorescence. To check this, use negative controls in which primary antibodies are omitted from the labeling process and observe samples with no fluorescent antibodies applied. Use fluorescent markers, with as widely separated excitation and emission spectra as possible, as secondary antibodies. A good typical example could be to use pairs of fluorescein isothiocyanate with Texas Red (excitation/emissions at around 495/520 and 589/615 nm, respectively) or Alexa Fluor 488 with Alexa Fluor 594 (excitation/emissions at around 495/519 and 590/617 nm, respectively $)^{16}$. The exact selection of markers will depend on the availability of primary antibodies. 


\section{BOX 1 | TERMINOLOGY}

Readers of the protocol, who are not entirely familiar with the used terminology or simply need clarification, can refer the text below. For more details, see references.

Median filtering is used to minimize errors when determining PPI values, especially in cases in which images have low ratio of specific fluorescence compared with the background nois ${ }^{14}$. Median filtering background reduction procedure estimates the value of background at each pixel by calculating the median value of $n X n$ square centered at this pixel, with $n$ being at least five times larger than the cluster size. The use of the large square size should guarantee that the median value reflects the level of background, which can then be subtracted from the image ${ }^{14}$.

Protein proximity index (PPI) is a quantitative measure of colocalization emphasizing the fact that colocalization occurs at the length scale of the resolution of the microscopes. Colocalization in dual-color fluorescence microscopic images results from two associated molecules of labeled proteins producing almost coincident resolution elements in each channel. In cross-correlation analysis, this coincidence corresponds to a fast-decaying peak, on top of a slow-decaying component of long-range correlations due to image heterogeneity and intracellular structures. The PPI method extracts the sharp peak to quantify colocalization more accurately. For ideal images free from background and noises, the numerical value of PPI is equal to the fraction of colocalized molecules ${ }^{14}$.

Background reduction is a computer-based procedure aimed at reducing noise in fluorescence microscopy images before quantification of colocalization by removing it (i.e., pixels of selected intensity values). Background reduction using advanced thresholding with selected ROI will select an area in the image background, which is dark, but not completely black, i.e., an area that has absorbed some fluorescence, although it was not supposed to. Pixel values within such areas can be considered as representative of the background. These pixels are subtracted from the image prior to calculation of coefficients.

Pearson's correlation coefficient (Rr) is frequently used to measure correlation between two variables (named after Karl Pearson). In quantitative colocalization analysis, it is applied to describe the correlation of the intensity distributions between channels. It considers the similarities between shapes while ignoring the intensities of signals $s^{3,7}$.

Overlap coefficient according to Manders $(R)$ is used to describe the overlap of signals in fluorescence microscopy images. First described by Erik Manders 5 . It is expected to be insensitive to sample photobleaching, efficiency of hybridization and camera quantum efficiency.

Overlap coefficients $k_{1}-k_{2}$ split the value of colocalization into two separate parameters; they are sensitive to the differences of the intensities between signals ${ }^{3,7}$.

Colocalization coefficients $m_{1}-m_{2}$ describe the contribution of each of two channels to the selected area on the image; they are insensitive to the intensities of fluorescence.

Colocalization coefficients $M_{1}-M_{2}$ are similar to $m_{1}-m_{2^{\prime}}$ but are applied for analyzing scattergram ROIs.

Scattergram (scatter plot) is a variation of mathematical diagram used for visualizing a set of data in the form of two variables. In quantitative colocalization analysis, it is used to display pixels of the analyzed image according to the pair of selected channels (for example, red/green).

ROI (region of interest) is a widely used abbreviation to define a specific area of the image selected for analytical purposes.

Creation of computer-simulated images. Pairs of green and red computer-simulated images with predefined values of colocalization for the red/green pair of channels can be used to create controls. With the help of BioSim simulation computer software (MATLAB source code is available at http://www.anes.ucla.edu/ wuyong/ biosim.zip), virtual 'molecules' were placed in a synthetic image. Further, each of them generated a PSF. The number of colocalized molecules was precisely controlled through the software. Synthetic images were created to resemble a 'blueprint' biological image, the intensity distribution of which is used as the probability distribution of the virtual 'molecules'. These images can be used to verify the steps of the procedure as well as serve as references by allowing users to compare the results of quantification obtained using them with the results obtained using users' custom images, if necessary (Supplementary Figs. 1-8).

Background reduction. Background in images is reduced either by median filtering or advanced thresholding. Median filtering combines two separate events, aimed at reducing discrete random noise and continuous background, respectively. Random noises, caused by electronic noise, dark current of detectors, scattered laser light and so on are detected and removed by protein proximity analyzer (PPA) software according to their discreteness: typically, they show themselves as 1-pixel-wide spikes in images. In contrast, continuous background, including out-of-focus light and smearedout intensity of the nonspecific labeling, is distributed with a longer length scale than the signal. In other words, the spatial variation of continuous background is smaller than the signal, and this is how it is detected and reduced by the software. Advanced thresholding is done by selecting a small area within the actual image background. Thereafter, computer software samples it according to the selected pair of channels, taking into consideration color components of the pixels. A pixel is considered red if its red component is above the minimum intensity value of 30 and green and blue are zero; green if its green component is above the minimum intensity value of 30 and red and blue are zero; and yellow if it has red and green components above the minimum intensity value of 30 and blue is zero. Pixels in this area with values that do not qualify as red, green or yellow are then subtracted from the image.

Quantifying colocalization. Next, the proposed PPI ${ }^{14}$ and a wellknown correlation coefficient ${ }^{3,4}$ are used to estimate colocalization. 
PPI is a quantitative measure of colocalization emphasizing the fact that colocalization occurs at the length scale of the resolution of the microscopes. Colocalization in dual-color fluorescence microscopic images results from two associated molecules of labeled proteins producing almost coincident resolution elements in each channel. The PPI method extracts the sharp peak in the correlation functions to quantify colocalization more accurately. For ideal images free from background and noise, the numerical value of PPI is equal to the fraction of colocalized molecules ${ }^{14}$. $\mathrm{Rr}$ is frequently used to measure correlation between two variables, and in quantitative colocalization analysis it is applied to describe the correlation of the intensity distributions between channels ${ }^{7}$.

\section{MATERIALS}

\section{EQUIPMENT}

$\triangle$ CRITICAL Specific equipment is not required for this protocol. Any confocal microscope capable of two-channel visualization of fluorescent markers and any computer that can run the required software packages are sufficient. Additional details about equipment used for fluorescence staining can be found in previously published protocols ${ }^{8,17}$.

- Confocal microscope (Zeiss or equivalent)

- Computer capable of running colocalization software packages (computers used in this protocol include a PC with a $3.2 \mathrm{GHz}$ AMD Phenom II X4 processor and a Mac with a $3.06 \mathrm{GHz} \mathrm{CPU}$ Intel Core i3 processor)

- PPA software (http://www.anes.ucla.edu/ wuyong/). The software is a free download but requires MATLAB runtime 7.10 (MathWorks).

- CoLocalizer Pro software version 2.5 (CoLocalization Research Software http://www.colocalizer.com/) The software is distributed commercially with free trial available upon request.

- Parallels Desktop software version 5 or later to run Windows and Macintosh software side by side (Parallels). The software is distributed commercially; free trial is available (optional).

\section{PROCEDURE}

\section{Background reduction}

1) Use either a local area network or removable media to transfer images from the confocal microscope to your computer.

2| To reduce background using median filtering and removing random noise, start up PPA software and open the image of your interest.

3| Under the main menu, select 'Analyze' and then 'Median Filter (Control + F)' (Protein Proximity Analyzer $\rightarrow$ Analyze $\rightarrow$ Median Filter; Fig. 2a).

4| In the opened dialog box, enter the number of pixels into the appropriate boxes to reduce continuous background and random noise. Use large and small squares for background and noise reduction, respectively. Use the number 32 as a large square size and the number 3 as a small square size (Fig. 2 b). $\triangle$ CRITICAL STEP Entered numbers represent large and small kernel sizes. The large kernel is a square around each pixel in which the median intensity value is used as an estimation of background. The kernel size should be at least three times larger than the typical cluster size (estimated by the sharp peak width of the autocorrelation function of the image; PPA software provides this function). Usually 32 pixels works well. The small kernel size should be 2 or 3 .

5| Click the ' $\mathrm{OK}$ ' button to execute the process of background reduction.

6| Alternatively, to reduce background using thresholding presets, start up CoLocalizer Pro software and either drag and drop the image you will be analyzing (from Step 1) to its main application window or open it through the Finder window. The software is designed to work with already merged images. Single-channel images can be merged using its Merge tool, if needed (Shift + Command $+M$ ).

7| Select background reduction settings among Auto (option A) or Manual modes using ROI selection (option B) a

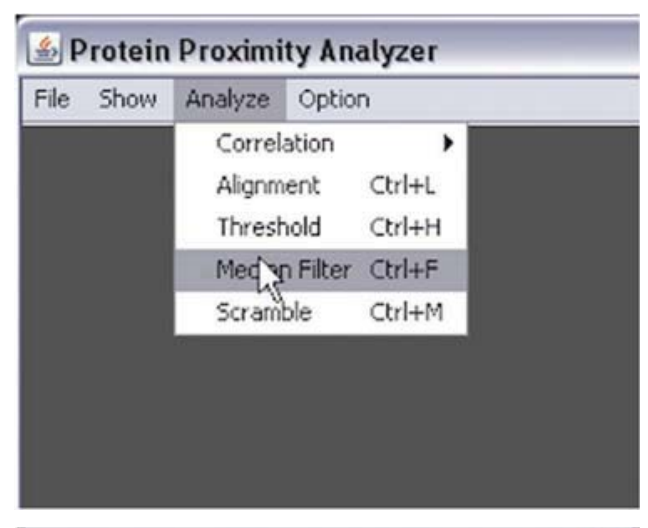

b

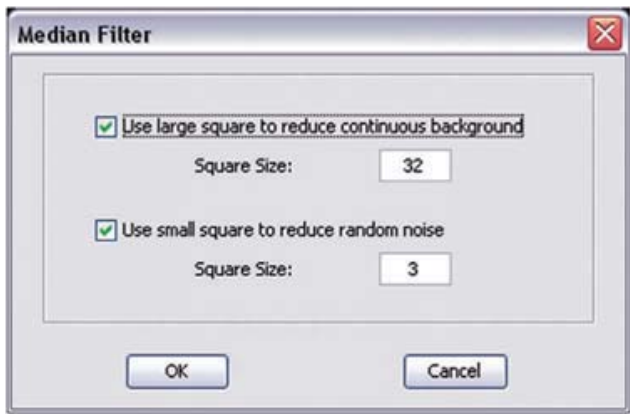

Figure 2 | Screenshots of the computer setup for using median filtering-based background reduction. After starting up the PPA software, open Median Filter under Analyze (a) and input the respective numbers of sizes for large and small squares (b) to reduce continuous background and random noise. 
or a threshold value (option C). Option B is capable of producing the most precise results because it can be adjusted to the unique pixel profiles of analyzed images. Although it requires a certain level of experience, even beginners can practice it without difficulty. Background reduction using manual threshold (option C) is the most approximate of all corrections because it is used primarily on the 'try-and-see' basis. Naturally, it easily introduces human bias in the quantification. This type of reduction is recommended to be used for the purpose of comparison with other types of correction, rather than on its own.

\section{(A) Reducing background in Auto mode}

(i) Click the 'Background' icon in the application toolbar or choose 'Background Correction' (Shift + Command + B) under the 'Tools' in the application menu bar.

(ii) In the opened 'Background Correction' window, select 'All' channels (Fig. 3).

(iii) Under the 'Auto' tab, select the preset closest to the pattern of the image being analyzed: 'Average Contrast and Fluorescence', 'Low Contrast' or 'Weak Fluorescence'; click 'Apply'. The 'Average Contrast and Fluorescence' option should be usable in the majority of cases (Fig. 3a). If the image is low contrast or the strength of fluorescence in it is too weak, the other respective presets should be applied.

I CAUTION Depending on the properties of analyzed images, the 'Low Contrast' and 'Weak Fluorescence' options may be too drastic and may remove too much pixel data.

(iv) Close the 'Background Correction' window by clicking 'Done'. As a result, the image in the main application window will appear slightly darkened because of the removal of the pixels determined by the preset values.

(v) After reduction, examine the image scattergram. If it shows that the black areas of subtracted pixels along its axes are too wide (more than $10 \%$ of the scattergram's width and height), background correction will need to be repeated using different settings, such as correction in the Manual mode using either the 'Threshold Value' or by selecting the ROI (see below). First, reset the image by selecting 'View' in the application menu bar and then choose 'Restore to Original'.

! CAUTION We recommend resetting the image to the original every time a different background reduction mode is chosen. It is also crucial not to resave the image as it may result in the loss of its original information, which is needed to achieve reproducible results.

$\triangle$ CRITICAL STEP Results of the background reduction procedure should be assessed by examining the image scattergram. It is necessary to clarify the results of background correction because visual representation of the images following background correction can be misleading.

(B) Reducing background in Manual mode using ROI selection

(i) Click the 'Background' icon in the application toolbar or choose 'Background Correction' under the 'Tools' in the application menu bar.

(ii) In the opened 'Background Correction' window, select 'All' channels.

(iii) Under the 'Manual' tab, click the 'Use Selected ROI' radio button (Fig. 3b).

(iv) By using either the 'Rectangular' or 'Oval' ROI selection tool (accessible under 'ROI' in the application menu bar), select a small area of approximately $10 \times 10$ pixels within the image background. When examining tissue sections, this area should be selected in the tissue background, which is dark but not completely black (i.e., it has absorbed some fluorescence). Pixel levels within such areas can be considered as representative of the background. When examining cells in culture, select a similarly small area that is free of cells. Click 'Apply' and then dismiss the 'Background Correction' window by clicking 'Done'. 
a

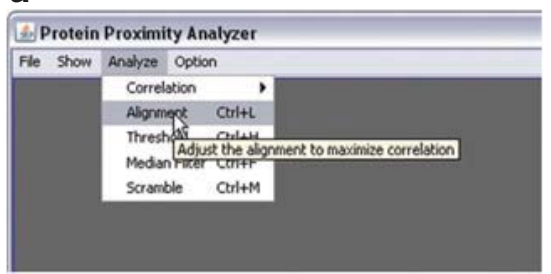

b

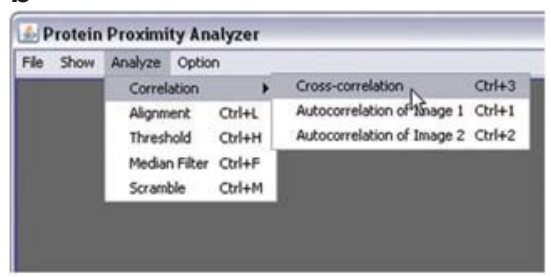

C

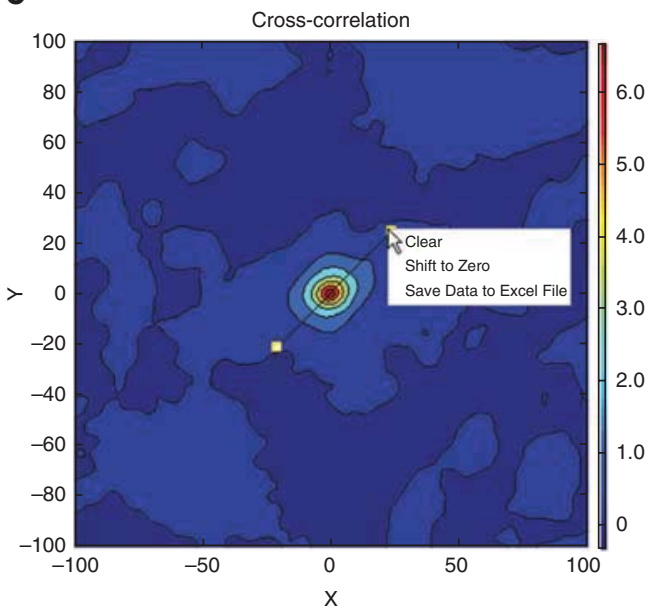

d

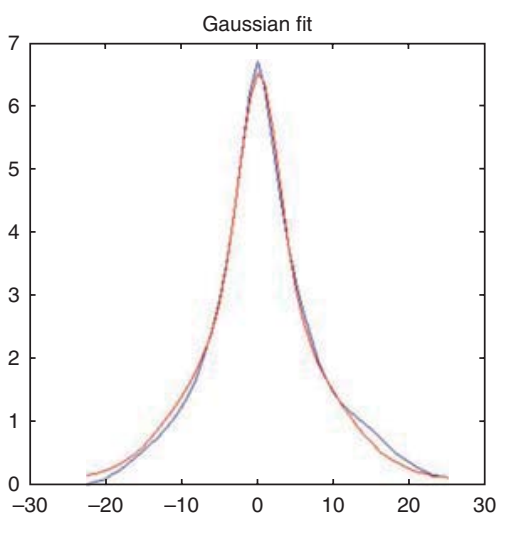

Figure 4 | Screenshots of the computer setup for performing PPI calculations. (a) First, image pairs need to be aligned. Next, cross-correlation and autocorrelation functions are determined (b) by drawing a straight line through the zero of the plot (c) and performing double Gaussian fitting (d).

(v) After reduction, examine the image scattergram.

$\Delta$ CRITICAL STEP Successful background reduction on images with colocalization will show the remaining pixels concentrating along the diagonal of the scattergram, whereas the majority of pixels located along the axes will be subtracted. The areas of subtracted pixels should not be too wide.

(C) Reducing background in Manual mode using a threshold value

(i) If any of the methods of reduction described above was used prior to this, reset the image by selecting 'View' from the application menu bar and then choose 'Restore to Original'. Click the 'Background' icon in the application toolbar or choose 'Background Correction' under the 'Tools' in the application menu bar.

(ii) In the opened 'Background Correction' window, select 'All' channels.

(iii) Under the 'Manual' tab, click the 'Use Threshold Value' radio button (Fig. 3c). This activates a slider to select the number of the pixels to remove. Choose somewhere between 20 and 40-this should produce the best results. Usually, 30 works well. Next, click 'Apply' and dismiss the 'Background Correction' window by clicking 'Done'.

$\triangle$ CRITICAL STEP Once they have been determined, the background reduction settings should be used consistently for all images in the study.

? TROUBLESHOOTING

\section{Quantification}

8| To quantify colocalization using PPI, use PPA software to open two gray scale images, one for each labeled protein, subjected to background reduction (as described in Steps 1-5).

9| Under the main menu, select 'Analyze' and then 'Alignment (Ctrl $+\mathrm{L}$ )' (Protein Proximity Analyzer $\rightarrow$ Analyze $\rightarrow$ Alignment). The image pairs are shifted to achieve maximal correlation using fast Fourier transform (Fig. 4a).

? TROUBLESHOOTING

10| Under the main menu, select 'Analyze' and then 'Correlation' (Protein Proximity Analyzer $\rightarrow$ Analyze $\rightarrow$ Correlation) to calculate the cross-correlation and autocorrelation functions (Fig. 4 b).

11| Each correlation function consists of a sharp peak located at the center and a surrounding shallow component. In the contour plot of the correlation function, draw a straight line through the zero of the plot, which follows the direction in which the shallow component drops slowly, and perform double Gaussian fit (Fig. 4c,d). After performing fitting for all correlation functions, derive PPI values from the ratio of correlation functions ${ }^{14}$.

$\triangle$ CRITICAL STEP The straight line where the double Gaussian fit is performed must follow the direction in which the shallow component drops slowest. On the contour plot, the shape of the shallow component is usually approximately elliptical. The slowest decaying direction follows the semi-major axis of the ellipse.

? TROUBLESHOOTING 
12| Alternatively, to quantify colocalization using coefficients, open the colocalization window of CoLocalizer Pro software with an image that has been subjected to background reduction (as described in Steps 6 and 7). Thereafter, select a pair of channels, for example, red/green ${ }^{7}$, according to which the coefficients should be calculated.

13| Calculate coefficients using either the whole image as an ROI (option A) or by selecting smaller areas with colocalization and performing calculations exclusively on them (option B). Coefficient numbers on smaller areas can be more precise as fewer pixels are included in the count.

(A) Calculating coefficients using whole image as a ROI

(i) Select the coefficients to be calculated by checking the appropriate individual boxes or check 'All' to select all. The software can calculate $\operatorname{Rr}, R$, overlap $k_{1}-k_{2}$ and colocalization $m_{1}-m_{2}$ coefficients.

$\triangle$ CRITICAL STEP Rr and $R$ coefficients have their standard values within different ranges: from -1.0 to 1.0 and from 0 to 1.0 , respectively. This should be taken into consideration when interpreting obtained results.

(ii) After calculating coefficients on the image ROI, calculate $M_{1}-M_{2}$ coefficients for the scattergram ROI to determine the contribution of each channel to the analyzed colocalization (optional).

(iii) Export the calculation results as text or Excel files for statistical analysis.

(iv) Save the results of calculations as session reports with coefficient numbers accompanied by the respective images in HTML and/or PDF format (optional).

(B) Calculating coefficients using ROI selections

(i) Under the software menu bar, choose 'ROI' and the type of selection that is most suitable for the shape of the area with colocalization to be analyzed, for example, rectangle (CoLocalizer Pro $\rightarrow$ ROI $\rightarrow$ Rectangle).

(ii) Select the area to examine. Try including as few pixels as possible that do not belong to the area with colocalization. The 'Polygon' and 'Lasso' tools usually provide the most accurate selection in regions with complex shapes.

$\Delta$ CRITICAL STEP It is possible to compare calculation results on images when using different selection tools as long as the pixel size of selected areas is the same.

(iii) Repeat Step $13 \mathrm{~A}(\mathrm{i}-\mathrm{iv})$.

14| (Optional) With the colocalization window opened, click the 'Reveal Pixels' tab to view where the colocalized pixels are located.

15 (Optional; continuing from Step 14) Under the opened tab, click the 'Colocalized' button to view the actually colocalized pixels in their image locations.

$\triangle$ CRITICAL STEP In addition to colocalized pixels, it is also possible to view pixels with other color values after selecting them on the image scattergram and then clicking the 'Select' button.

\section{? TROUBLESHOOTING}

Troubleshooting advice can be found in Table 1.

TABLE 1 | Troubleshooting table.

\begin{tabular}{|c|c|c|c|}
\hline Step & Problem & Possible reason & Solution \\
\hline 7 & $\begin{array}{l}\text { Scattergram shows an exces- } \\
\text { sive number of removed pixels }\end{array}$ & $\begin{array}{l}\text { The mode used for background reduc- } \\
\text { tion is not suitable for the image }\end{array}$ & $\begin{array}{l}\text { If reduced in the Auto mode, switch to Manual and try } \\
\text { using 'ROI selection'. } \\
\text { If corrected using Selected ROI, choose a new ROI in } \\
\text { the different background portion of the image. } \\
\text { If corrected using 'Threshold Value', decrease the number } \\
\text { of intensity levels to be subtracted (for example, 10) }\end{array}$ \\
\hline 9 & $\begin{array}{l}\text { Shift values needed to align } \\
\text { the image pair are too large } \\
\text { (more than } 10 \text { pixels for } \\
\text { typical images) }\end{array}$ & $\begin{array}{l}\text { Image pair has very low colocaliza- } \\
\text { tion; the maximum cross-correlation } \\
\text { value is merely due to random fluc- } \\
\text { tuation and has no meaning }\end{array}$ & $\begin{array}{l}\text { Do not adjust alignment. Go directly to calculate and } \\
\text { show correlation functions. If the cross-correlation } \\
\text { function lacks a sharp peak, the colocalization is } \\
\text { almost zero }\end{array}$ \\
\hline 11 & Quality of fit is unsatisfactory & $\begin{array}{l}\text { The initial values of fitting param- } \\
\text { eters are poor estimates (the initial } \\
\text { values need to be reasonably good } \\
\text { estimates for fitting to converge } \\
\text { successfully and quickly) }\end{array}$ & $\begin{array}{l}\text { Use default values by clicking the 'Default Value' but- } \\
\text { ton in the 'Line Scan Analysis' window. Alternatively, } \\
\text { input initial values according to your own estimation }\end{array}$ \\
\hline
\end{tabular}


a

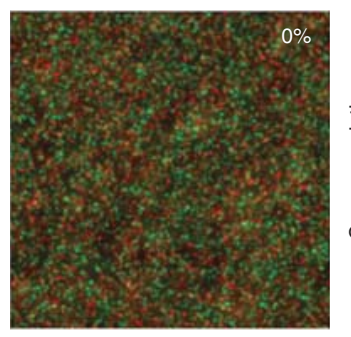

b

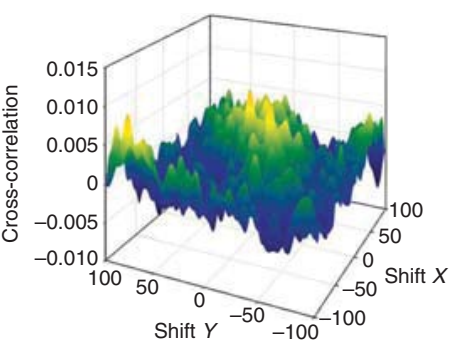

C

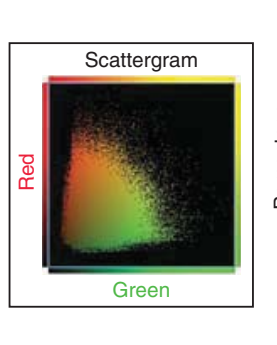

d

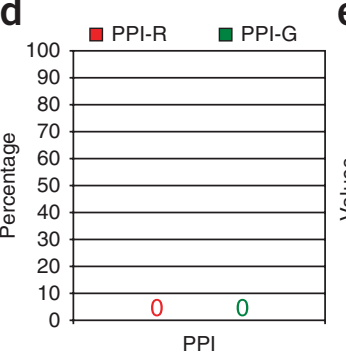

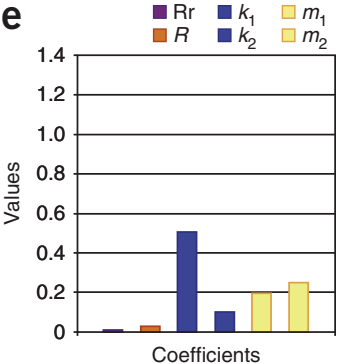

Figure 5 | Quantification of colocalization using computer-simulated dual-color images with predefined colocalization and without any background or nonspecific fluorescence. We used the PPI, $\operatorname{Rr} R, k_{1}-k_{2}$ and $m_{1}-m_{2}$ to estimate colocalization. (a) Quantification of the image with $0 \%$ colocalization. $3 \mathrm{D}$ plot does not show any single sharp peak indicating colocalization (b), but 2D scattergram reveals only widely scattered pixels (c). (d) PPI numbers are perfect 0 for both red and green channels. (e) The values of all coefficients are very low.

\section{TIMING}

Steps 1-5, Background reduction using median filtering: 3-5 min (for an image of $512 \times 512$ pixels, using a PC with a 3.2 GHz AMD Phenom II X4 processor)

Steps 6 and 7, Background reduction by thresholding: $<1$ min when performing threshold-based reduction in Auto mode (Step 7A) (for an image of $512 \times 512$ pixels, using a Mac with a $3.06 \mathrm{GHz}$ CPU Intel Core i3 processor). In Manual mode with ROI selection (Step 7B), the timing may be longer because of the need to find the appropriate area within the image background, but it should not exceed 5 min in most cases.

Steps 8-11, Quantifying colocalization using PPI: <1 min (for an image of $512 \times 512$ pixels, using a PC with a $3.2 \mathrm{GHz}$ AMD Phenom II $X 4$ processor)

Steps 12 and 13, Quantifying colocalization using coefficients: <1 min when calculating coefficients on the whole image as an ROI (Step 13A) (for an image of $512 \times 512$ pixels, using a Mac with a $3.06 \mathrm{GHz}$ CPU Intel Core i3 processor). With a smaller ROI (Step 13B), the timing may be longer because of the need to carefully outline the area with colocalization with a minimum of surrounding pixels, but it should not exceed 3 min in most cases.

The entire procedure should not exceed approximately 15-30 min.

\section{ANTICIPATED RESULTS}

Quantification using simulated images with predefined colocalization values in the absence of background and nonspecific fluorescence

To illustrate the applicability of the protocol, we start with figures (Figs. 5-8) showing computer-simulated images with varying degrees of colocalization (see Experimental Design and Supplementary Figs. 1-8). The images have predefined values of colocalization for the red/green pair of channels (0\%, 25\%, 50\% and 75\%, Figs. 5a-8a) and are free of any background and nonspecific fluorescence; thus none of the background reduction steps need to be applied. The images are accompanied by 3D plots showing cross-correlation (Figs. 5b-8b) and 2D scattergrams showing the distribution of pixels according to the selected pair of channels (Figs. 5c-8c). PPI ${ }^{14}, R r, R, k_{1}-k_{2}$ and $m_{1}-m_{2}$ were used to quantify colocalization $^{3,4}$. As expected, all calculations showed clear responses to the gradual increase of the degree of colocalization. The PPI for the red/green pair of channels with $0 \%, 25 \%, 50 \%$ and $75 \%$ of colocalization was $0 \% / 0 \%, 23 \% / 24 \%, 50 \% / 50 \%$ and $74 \% / 73 \%$, respectively (Figs. $5 \mathbf{d}-\mathbf{8 d}$ ). Accordingly, the values of all coefficients increased together with the increase in the degree of colocalization. The changes of the $\operatorname{Rr}$ coefficient for the $0 \%, 25 \%, 50 \%$ and $75 \%$ images were $0.01,0.22,0.47$ and

a

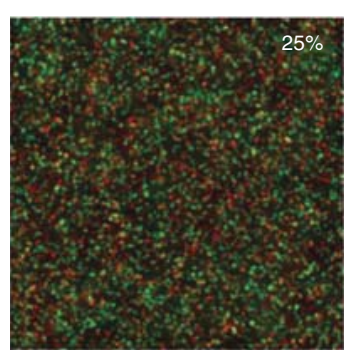

b

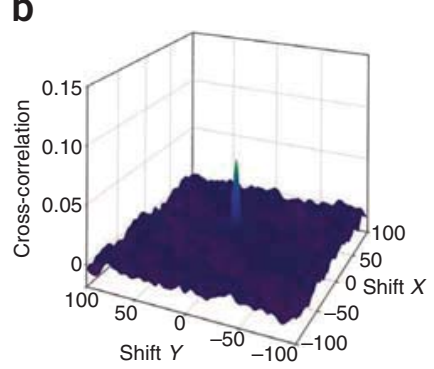

C

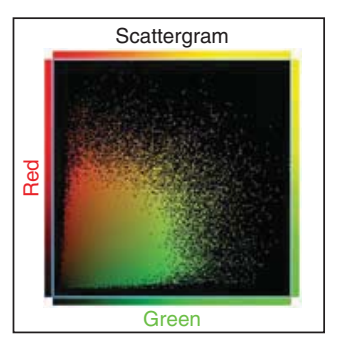

d

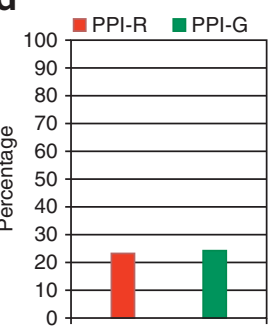

PPI

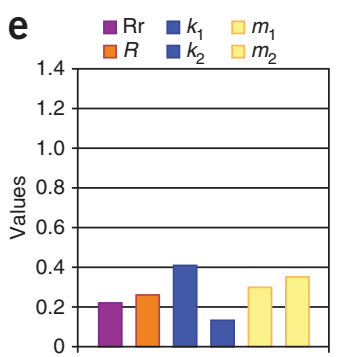

Coefficients

Figure 6 | Quantification of colocalization using computer-simulated dual-color images with predefined colocalization and without any background or nonspecific fluorescence. We used the PPI, Rr, $R, k_{1}-k_{2}$ and $m_{1}-m_{2}$ to estimate colocalization. (a) Quantification of the image with $25 \%$ colocalization. Note the appearance of a small peak at the center of the 3D plot (b), suggesting some colocalization, whereas pixels on 2D scattergram are still scattered very widely (c). Both PPI (d) and coefficients (e) started to show some response to the increased colocalization. 
a

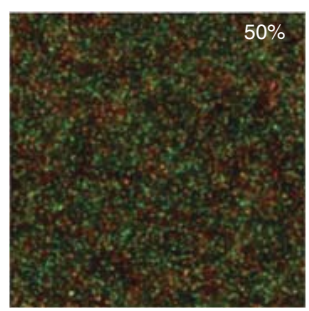

b

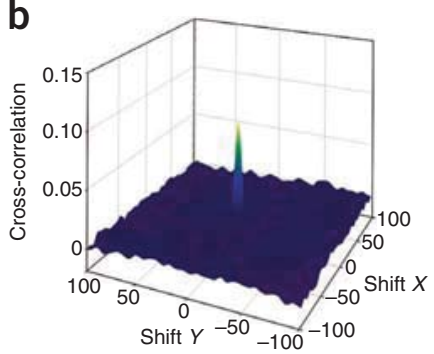

C

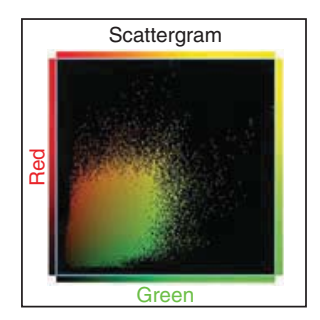

d

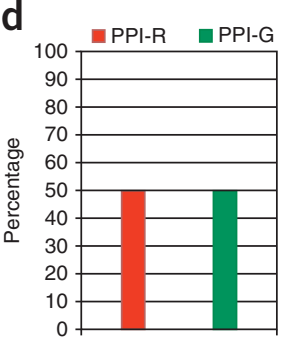

PPI

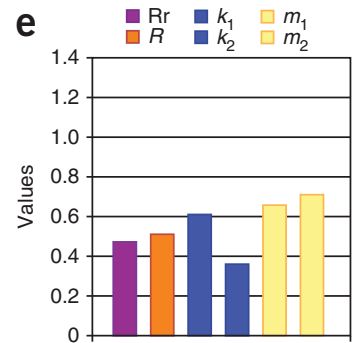

Coefficients

Figure 7 | Quantification of colocalization using computer-simulated dual-color images with predefined colocalization and without any background or nonspecific fluorescence. We used the PPI, Rr, $R, k_{1}-k_{2}$ and $m_{1}-m_{2}$ to estimate colocalization. (a) Quantification of the image with $50 \%$ colocalization. (b) The peak at the center of the 3D plot became taller and (c) pixels on 2D scattergram started moving toward its center. Both PPI (d) and coefficients (e) further increased their values.

0.70 , respectively. The changes of the $R$ coefficient for the $0 \%, 25 \%, 50 \%$ and $75 \%$ images were $0.03,0.26,0.51$ and 0.72 , respectively. The gap between the $k_{1}$ and $k_{2}$ values for the $0 \%, 25 \%, 50 \%$ and $75 \%$ images shortened, indicating an increase in colocalization, from 0.40 to $0.28,0.25$ and 0.12 , respectively. The $m_{1}-m_{2}$ coefficients for the $0 \%, 25 \%, 50 \%$ and $75 \%$ images rose from $0.20 / 0.25$ to $0.30 / 0.36,0.66 / 0.71$ and $0.81 / 0.90$, respectively, similarly indicating an increase in colocalization (Figs. 5e-8e).

\section{Quantification using real biological images with background intact}

Figure 9 gives an example of a typical image with colocalization by showing dual staining for ryanodine receptors (red) and estrogen receptor- $\alpha$ (green) in isolated mouse cardiac myocytes ${ }^{14}$ (Fig. 9a). The image shown has very low values of colocalization (close to zero) deliberately chosen to illustrate the impact that background noise makes, especially in the case of the images with low colocalization, and to allow a meaningful computer simulation on the images described below. Expectedly, 3D plot and 2D scattergram did not show either any sharp peak located at the center of the plot (Fig. 9b) or pixels concentrated at the diagonal of the scattergram (Fig. 9c). Instead, pixels representing background can be seen alongside the $x$ and $y$ axes (Fig. 9c). The lack of the background reduction procedure resulted in overestimation of colocalization on this image, especially clearly seen for coefficient calculations: $\operatorname{Rr}$ was 0.36 (according to -1.0 to 1.0 scale), $R$ was 0.43 (according to $0-1.0$ scale), the gap between $k_{1}$ and $k_{2}$ values constituted 0.43 , whereas $m_{1}-m_{2}$ was $0.85 / 0.81$ (Fig. 9e). PPI values were $8 \% / 6 \%$ (according to $100 \%$ scale; Fig. 9c) and overestimated colocalization as well, albeit to a smaller degree.

\section{Quantification using median filtering-based background reduction followed by PPI estimation}

Thereafter, we created a set of computer-simulated images modeled on a real biological image (shown in Fig. 9) with predefined $0 \%, 25 \%, 50 \%$ and $75 \%$ of colocalization and added some background noise (Fig. 10a-d). When creating these images, their intensity was initially set to zero. Protein clusters, treated as point sources, were randomly positioned according to their biological model images; in a simulated image, the probability of protein occupying a pixel is proportional to the intensity of the corresponding pixel in its biological counterpart. Each of the clusters generated an intensity distribution according to a Gaussian PSF. The degree of colocalization was precisely controlled by knowing the exact number of clusters generated by computer software. Specifically labeled clusters were distinguishable from the nonspecifically labeled ones by being substantially brighter. We generated random noise by the absolute value of Gaussian random numbers. We then performed background reduction and PPI estimation on these images. We reduced background by using a special median filtering

a

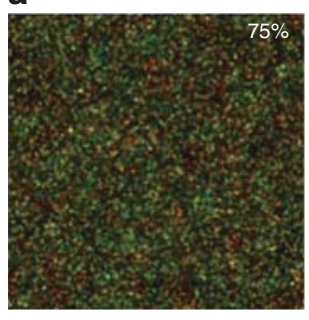

b

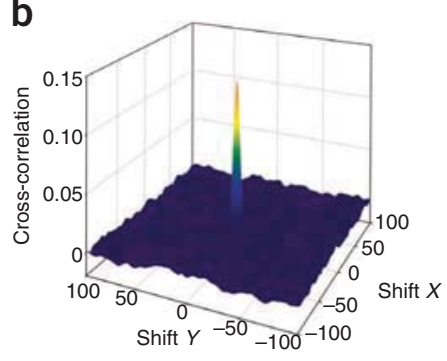

C

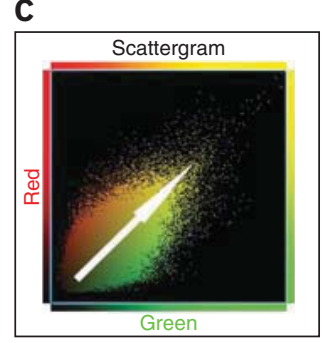

d

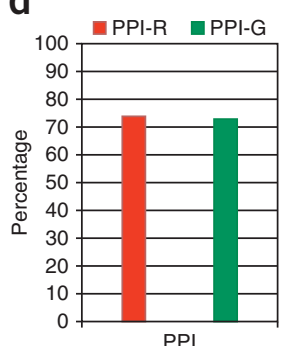

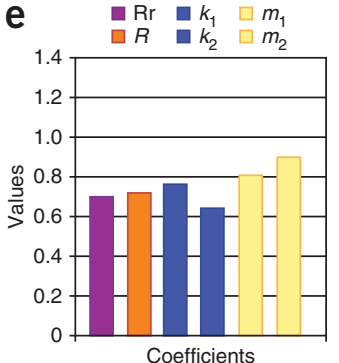

Figure 8 | Quantification of colocalization using computer-simulated dual-color mages with predefined colocalization and without any background or nonspecific fluorescence. We used the PPI, Rr, $R, k_{1}-k_{2}$ and $m_{1}-m_{2}$ to estimate colocalization. (a) Quantification of the image with $75 \%$ colocalization. Note a substantial increase in the peak height at the center of the 3D plot (b) and an increase of the representation of yellow (colocalized) pixels along the diagonal (arrow) on the 2D scattergram (c). PPI (d) and coefficients (e) increased their values even more. 
a

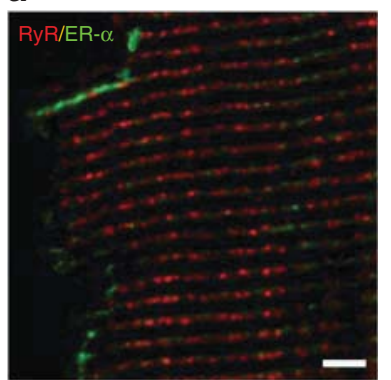

b

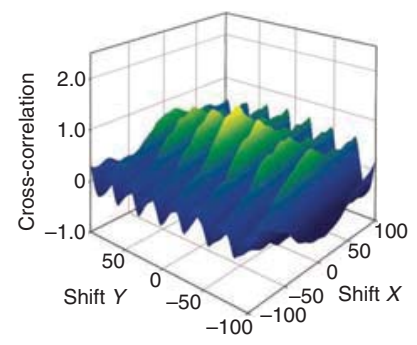

C

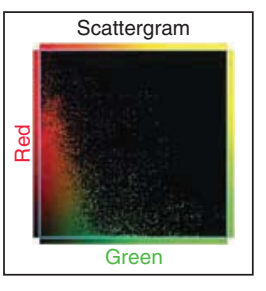

d

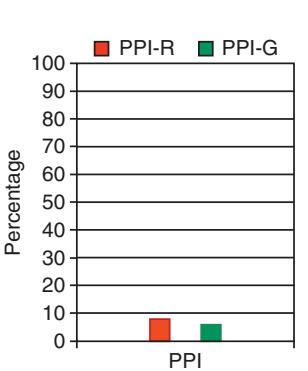

e

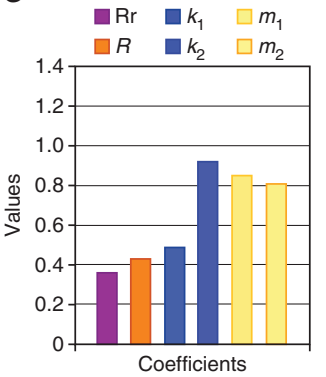

Figure 9 | Quantification of colocalization using a real biological image with nonreduced background. Dual staining for ryanodine receptor (RyR; red fluorescence) and estrogen receptor- $\alpha$ (ER- $\alpha$; green fluorescence) (a) in isolated mouse cardiac myocyte with almost zero colocalization (scale bar, $5 \mu \mathrm{m}$ ) is accompanied by 3D plot (b) and 2D scattergram (c). Note the absence of the peak at the center of the 3D plot and pixels on the diagonal in 2D scattergram. We used the PPI, $\mathrm{Rr}, R, k_{1}-k_{2}$ and $m_{1}-m_{2}$ to estimate colocalization. The presence of background clearly contributes to the overestimation of colocalization on the basis of the PPI (d) and coefficient (e) numbers. The image shown is used to create the computer-simulated images in Figures 10 and 11.

procedure by setting separate large and small square sizes to control both continuous image background and random noise in $\mathrm{it}^{14}$. As described above, median filtering combines two separate events, corresponding to small and large kernel sizes as user inputs in the PPA software. In small kernel median filtering, intensity of each pixel is replaced by the median of the kernel, which eliminates the spikes of random noises. In large kernel median filtering, the background level in the kernel is estimated by its median and then subtracted from the pixel intensity value. Further, PPI values are calculated. 3D plots showed a gradual increase in the size of the peak at the center of the plot (Fig. 10e-h). Accordingly, PPI numbers revealed gradual increases in their values as well (Fig. 10i-l), suggesting that both the background reduction procedure and PPI
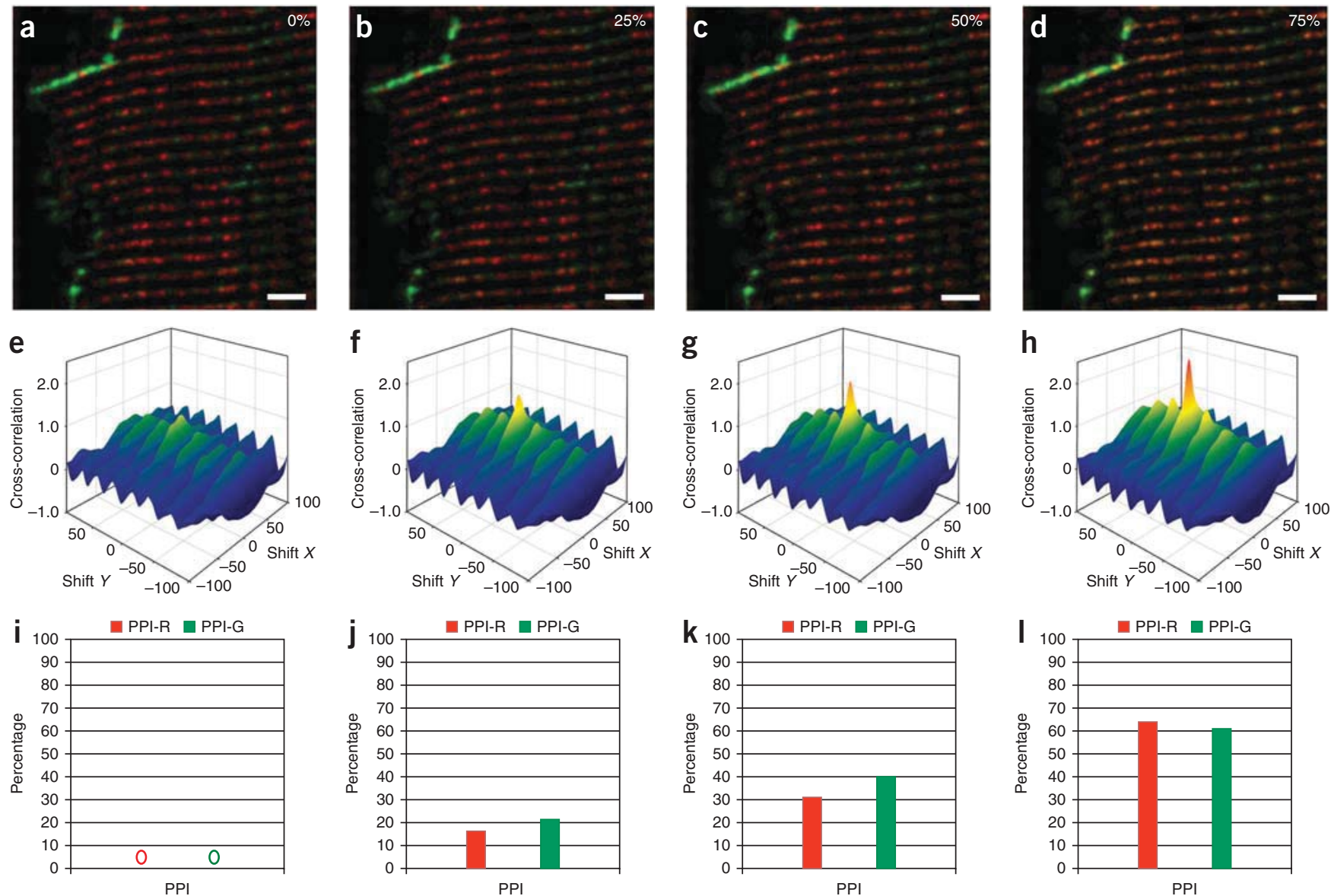

PPI

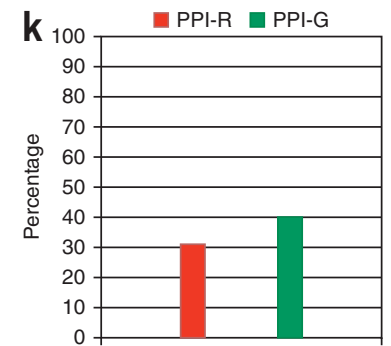

PPI

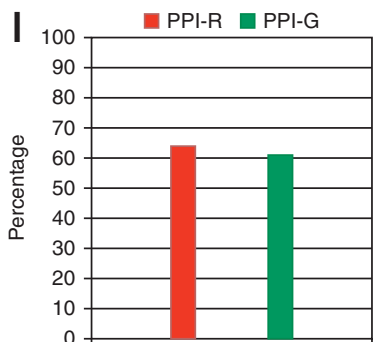

PPI

Figure 10 | Quantification of colocalization on a set of computer-simulated images modeled on a real biological image shown in Figure 9. (a-h) Images have predefined $0 \%, 25 \%, 50 \%$ and $75 \%$ values of colocalization (a-d) with added background noise (scale bars, $5 \mu \mathrm{m}$ ) and are accompanied by their respective 3D plots (e-h). Images were processed using median filtering background reduction procedure. PPI values were calculated to estimate colocalization. (i-l) Background reduction ensured a gradual increase in PPIs, clearly reflecting the respective increase in predefined colocalization. 
calculations worked very well. Notably, the image with zero colocalization had perfect zero values of PPI for both red and green channels. For images with $25 \%, 50 \%$ and $75 \%$ colocalization, PPI values at higher degrees of colocalization were, as expected, a little less precise compared with those obtained for computer-simulated images with no background and nonspecific fluorescence, but they were still very close to the predefined values: $16 \% / 21 \%$, $31 \% / 40 \%$ and $64 \% / 61 \%$, respectively.

\section{Quantification using median filtering- based background reduction followed by coefficient estimations}

We also compared whether the calculation of coefficients can be used with images after the median filtering procedure. The results of $\mathrm{Rr}$ calculations were as follows: $0.10,0.21,0.49$ and 0.71 (compared with $0.04,0.24,0.56$ and 0.8 for images after thresholdbased background reduction). The results of $R$ calculations were as follows: $0.44,0.49,0.47$ and 0.46 (compared with $0.46,0.51,0.57$ and 0.62 ). The $k_{1}-k_{2}$ gap was as follows: $0.83,0.52,0.46$ and 0.33 (compared with $0.71,0.39,0.29$ and 0.21 ). Finally, the values of the $m_{1}$ and $m_{2}$ coefficients were 0.11 and $0.12,0.27$ and $0.26,0.47$ and 0.39 , and 0.79 and 0.82 (compared with 0.19 and 0.18 , 0.33 and $0.39,0.58$ and 0.49 , and 0.83 and 0.64 ; Table 2 ). These results indicate compatibility of images processed using median filtering- and threshold-based background reduction tools for quantification of colocalization using PPI and coefficient estimations (with the exception of the $R$ coefficient).

\section{Quantification using threshold-based background reduction followed by PPI estimation}

We then examined how the threshold-based background reduction procedure affects the results of PPI estimation and found the following values for images with $0 \%, 25 \%, 50 \%$ and $75 \%$ colocalization: $0 \% / 0 \%, 23 \% / 19 \%, 37 \% / 44 \%$ and $67 \% / 61 \%$ (compared with $0 \% / 0 \%, 16 \% / 21 \%, 31 \% / 40 \%$ and $64 \% / 61 \%$ for images after median filtering (Table 2 )). These results suggest that images after threshold-based background reduction procedure can be reliably used for PPI estimation as well.

\section{Quantification using threshold-based background reduction followed by coefficient estimations}

To compare the results of background reduction using median filtering and PPI estimation with threshold-based reduction and coefficient calculations, we used the same set of images with predefined $0 \%, 25 \%, 50 \%$ and $75 \%$ of colocalization and added background noise (Fig. 11a-d). Background reduction was performed by selecting a small (10 × 10 pixels) area within the actual image background and using CoLocalizer Pro software to sample it according to the selected red/green pair of channels, taking color components into consideration. Pixels were evaluated according to the minimum intensity levels for the selected channels as described in the Experimental Design section. Determined values of the selected area were then automatically subtracted from the image. The area of selected pixels can vary from $5 \times 5$ to $10 \times 10$ pixels but should always remain the same for all images in the study to ensure comparability of results. Furthermore, we calculated Rr, $R$, the overlap coefficients $k_{1}-k_{2}$ and the colocalization coefficients $m_{1}-m_{2}$. 2D scattergrams show the distribution of pixels according to the red/green pair of channels (Fig. 11e-h). Calculation results indicate that, in general, the increase of predefined colocalization is well reflected by the respective increase in the values of calculated coefficients. However, not all coefficients were equally useful. The most applicable is Rr; it changed its values from 0.04 to $0.24,0.56$ and 0.80 . The least applicable is $R$; it changed from 0.46 to $0.51,0.57$ and 0.62 (Fig. 11i-l). These findings suggest that the threshold-based background reduction procedure we used is also suitable, and that it produces the best results for $k_{1}-k_{2}, m_{1}-m_{2}$ and especially for the Rr coefficient; however, it should be applied very cautiously when determining the values of $R$ coefficient, as even the background 

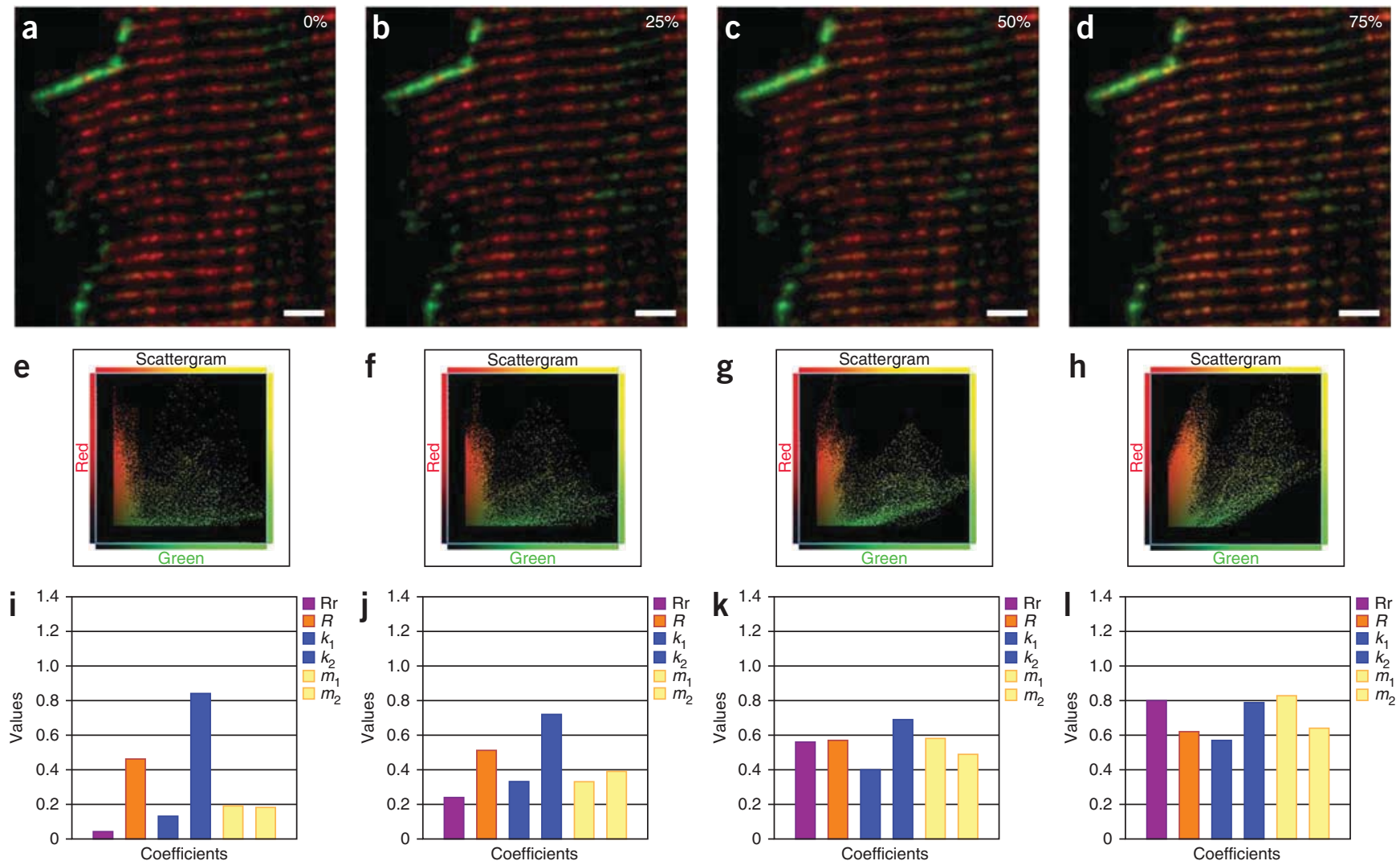

Figure 11 | Quantification of colocalization on a set of computer-simulated images modeled on a real biological image shown in Figure 9. (a-h) Images have predefined $0 \%, 25 \%, 50 \%$ and $75 \%$ values of colocalization (a-d) with added background noise (scale bars $5 \mu \mathrm{m}$ ) and are accompanied by their respective scattergrams (e-h). We used the PPI, $\mathrm{Rr}, R, k_{1}-k_{2}$ and $m_{1}-m_{2}$ to estimate colocalization. Coefficient values were obtained following thresholdbased background reduction procedure, as indicated by the areas of removed pixels along the $x$ and $y$ axes of the scattergram. (i-l) Note that, although all coefficients show a gradual increase, the values of $\mathrm{Rr}$ coefficient are the most responsive.

reduction we used could not circumvent its limitations. These observations are in agreement with the known fact that the $R$ coefficient is very sensitive to background noise ${ }^{6}$ and is, overall, less useful than $\operatorname{Rr}^{18}$. As a rule, images that have had their backgrounds reduced using median filtering should be quantified using PPI, whereas images that have had their backgrounds reduced using thresholding should be quantified using Rr. If any questions about results arise and/or there is a need to double-check the obtained numbers, then images processed by median filtering can be analyzed with the help of Rr and PPI can be used for the threshold-processed ones.

\section{Quantification of colocalization on a real biological image following background reduction}

Figures 12-14 show the results of background reduction and quantification of colocalization on a real biological image (0.G.-Z. and V.Z., unpublished data). The image depicts colocalization of the platelet-activating factor receptor (red fluorescence) and
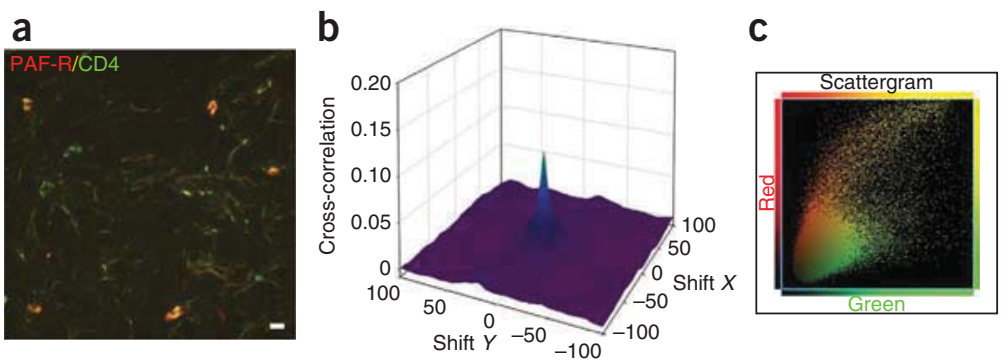

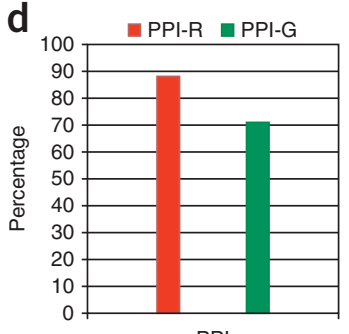

PPI

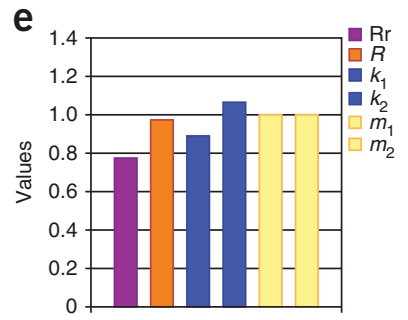

Coefficients

Figure 12 | Quantification of colocalization on a real unprocessed biological image showing dual staining for platelet-activating factor receptor (PAF-R; red fluorescence) and CD4 (green fluorescence) on macrophages in conjunctiva. We used the PPI, Rr, $R, k_{1}-k_{2}$ and $m_{1}-m_{2}$ to estimate colocalization. The image has moderate levels of colocalization and background (a) (scale bar, $10 \mu \mathrm{m}$ ), as indicated by 3D plot (b) and 2D scattergram (c). (d) PPI is $88 \%$ and $71 \%$ for red and green channels, respectively. (e) $\operatorname{Rr}$ and $R$ coefficients are 0.77 and 0.97 , respectively. 
Figure 13 Quantification of colocalization on the image shown in Figure 12 after median filtering background reduction and PPI estimations. (a) The image looks slightly darkened. Scale bar, $10 \mu \mathrm{m}$. (b) Note a substantially taller peak at the center of the 3D plot. (c) PPI values changed to $83 \%$ and $78 \%$ for red and green channels, respectively, indicating the impact of median filtering. PAF-R, plateletactivating factor receptor. a

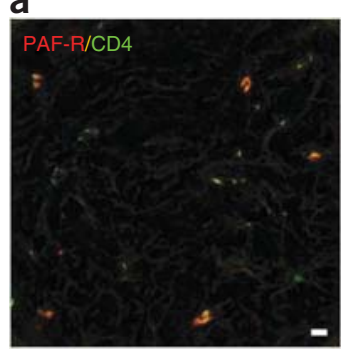

b

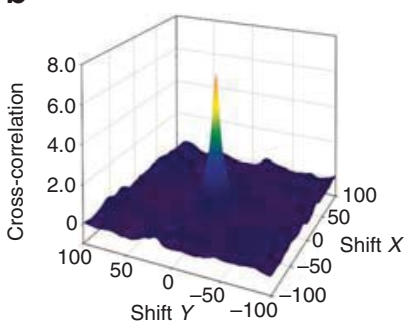

C

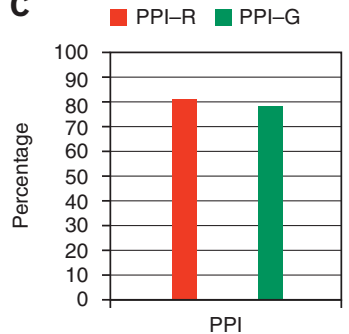

CD4 (green fluorescence) on macrophages in the rat conjunctiva. It has moderate levels of colocalization and background. We first calculated PPI and a set of coefficients on the intact image without performing any background reduction (Fig. 12a-e). Next, we processed the image to reduce the contribution of background by using median filtering- (Fig. 13a-c) and threshold-based correction (Fig. 14a-c) and quantified by calculating the values of PPI (Fig. 13a-c) and a set of coefficients (Fig. 14a-c). Reduction of the background using median filtering resulted in the change of PPI values for both red (88\% before and $83 \%$ after) and green channels (71\% before and $78 \%$ after), suggesting that quantification of colocalization using PPI on intact biological images would have been wrong, i.e., misinterpreted by approximately $5-7 \%$. Reduction of the background using threshold-based correction showed a decrease in the values of all coefficients following quantification. We observed the largest decrease in the values of coefficients for $\operatorname{Rr}(0.77$ before and 0.53 after, $31 \%$ decrease, which is expected as a result of background reduction). We observed the smallest decrease for $R\left(0.97\right.$ and $0.96,1 \%$ decrease). The values of $k_{1}-k_{2}$ and $m_{1}-m_{2}$ coefficients changed from $0.89 / 1.06$, gap $=0.17-0.51 / 0.86$, gap $=0.35$ and from 1.0 to 0.76 and $1.0-0.72$, respectively. Similarly, these results suggested that quantification of colocalization using a set of coefficients on intact biological images would have resulted in misinterpretation of colocalization. Comparison of the response of coefficients to threshold-based reduction using this image with that obtained using computer-simulated images suggested good usability of $k_{1}-k_{2}, m_{1}-m_{2}$ and, especially, the Rr coefficient, whereas the $R$ coefficient appeared to be of limited use. Thus, considering compatibility of these quantification tools (Table 2), they can be used to double-check each other to ensure reliability of obtained results. Following background reduction, spatial correlation of fluorescent markers can be quantified using PPI and Rr estimations. PPI can be used to obtain separate values of each channel to characterize the respective contribution of each protein, whereas $\mathrm{Rr}$ can be used to determine the overall degree of colocalization. It should be mentioned that, in the given example, threshold-based correction was performed in the Manual mode using ROI selection, as the image background contained a relatively small amount of surrounding tissue and selecting a suitable ROI was easy. In the case of the larger amount of tissue in the background, using the Auto mode with image pattern-based presets would have been a more appropriate choice (see TROUBLESHOOTING).
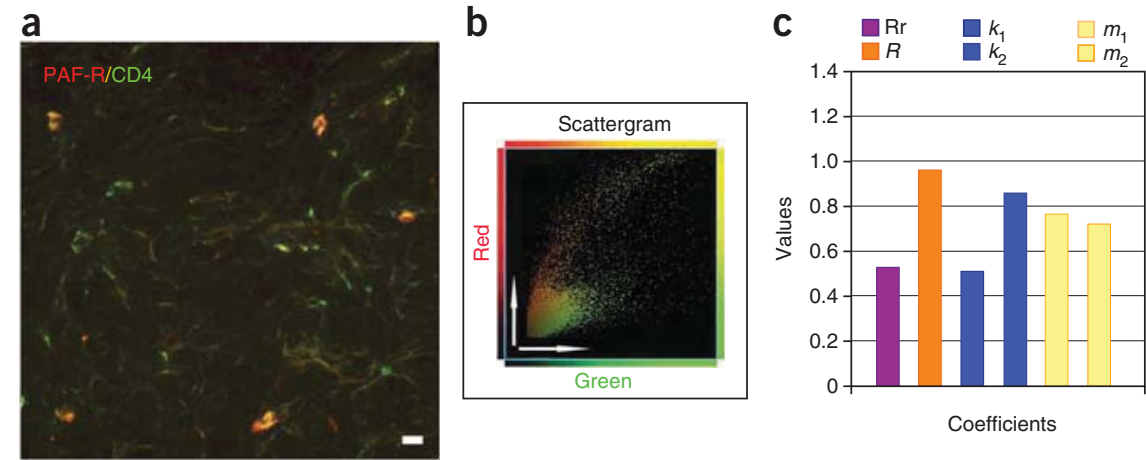

Figure 14 | Quantification of colocalization on the image shown in Figure 12 after threshold-based background reduction and coefficient estimations. (a) The image appears almost unchanged. Scale bar, $10 \mu \mathrm{m}$. (b) Note the areas of removed pixels along $x$ and $y$ axes (arrows). (c) The values of $\operatorname{Rr}$ and $R$ coefficients changed to 0.53 and 0.96 , respectively, indicating the impact of background thresholding. PAF-R, platelet-activating factor receptor.
Note: Supplementary information is available via the HTML version of this article.

ACKNOWLEDGMENTS This work was partially supported by National Institutes of Health grant no. HL088640 and the American Heart Association Postdoctoral Fellowship no. 10P0ST4230081. We thank M. Celio (Fribourg University) for help in organizing the study.

AUTHOR CONTRIBUTIONS V.Z. conceived and organized the study, designed and conducted experiments and wrote the paper; Y.W. conducted experiments and contributed to writing, 0.G.-Z. designed and conducted experiments and contributed to writing; and E.S. helped to organize the study.

COMPETING FINANCIAL INTERESTS The authors declare no competing financial interests.
Published online at http://www.natureprotocols.com/.

Reprints and permissions information is available online at http://www.nature. com/reprints/index.html.

1. Kuriyan, J. \& Eisenberg, D. The origin of protein interactions and allostery in colocalization. Nature 450, 983-990 (2007).

2. Konig, P. et al. FRET-CLSM and double-labeling indirect immunofluorescence to detect close association of proteins in tissue sections. Lab. Invest. 86, 853-864 (2006).

3. Smallcombe, A. Multicolor imaging: the important question of co-localization. BioTechniques 30,1240-1242, 1244-1246 (2001).

4. Zinchuk, V. \& Grossenbacher-Zinchuk, 0. Recent advances in quantitative colocalization analysis: focus on neuroscience. Prog. Histochem. Cytochem. 44, 125-172 (2009). 
5. Manders, E.M.M., Verbeek, F.J. \& Aten, J.A. Measurement of co-localization of objects in dual-colour confocal images. J. Microsc. 169 , 375-382 (1993).

6. Bolte, S. \& Cordelieres, F.P. A guided tour into subcellular colocalization analysis in light microscopy. J. Microsc. 224, 213-232 (2006)

7. Zinchuk, V. \& Zinchuk, 0. Quantitative colocalization analysis of confocal fluorescence microscopy images. Curr. Protoc. Cell Biol. Chapter 4 Unit 4 19 (2008).

8. French, A.P., Mills, S., Swarup, R., Bennett, M.J. \& Pridmore, T.P. Colocalization of fluorescent markers in confocal microscope images of plant cells. Nat. Protoc. 3, 619-628 (2008).

9. Waters, J.C. Accuracy and precision in quantitative fluorescence microscopy. J. Cell Biol. 185, 1135-1148 (2009).

10. Ono, M. et al. Quantitative comparison of anti-fading media for confocal laser scanning microscopy. J. Histochem. Cytochem. 49, 305-312 (2001).

11. Landmann, L. \& Marbet, P. Colocalization analysis yields superior results after image restoration. Microsc. Res. Tech. 64, 103-112 (2004).
12. Zinchuk, V., Zinchuk, 0., Akimaru, K., Moriya, F. \& Okada, T. Ethanol consumption alters expression and colocalization of bile salt export pump and multidrug resistance protein 2 in the rat. Histochem. Cell Biol. 127, 503-512 (2007).

13. Shaw, P. \& Rawlins, D.J. The point spread function of confocal microscope: its measurement and use in deconvolution. J. Microsc. 163, 151-165 (1991).

14. Wu, Y. et al. Quantitative determination of spatial protein-protein correlations in fluorescence confocal microscopy. Biophys. J. 98, 493-504 (2010).

15. Lippincott-Schwartz, J. \& Manley, S. Putting super-resolution fluorescence microscopy to work. Nat. Methods 6, 21-23 (2009).

16. Haugland, R.P. The Handbook: A Guide to Fluorescent Probes and Labeling Technologies 10th edn (Invitrogen, Inc., 2005).

17. Schneider Gasser, E.M. et al. Immunofluorescence in brain sections: simultaneous detection of presynaptic and postsynaptic proteins in identified neurons. Nat. Protoc. 1, 1887-1897 (2006).

18. Adler, J. \& Parmryd, I. Quantifying colocalization by correlation: the Pearson correlation coefficient is superior to the Mander's overlap coefficient. Cytometry A 77, 733-742 (2010). 